\title{
Polarization-Insensitive Reflective Metasurfaces for Highly Efficient Generation of OAM Beams
}

\author{
Xudong Bai* \\ R\&D Center, Shanghai Aerospace Electronics Company Ltd., China Academy of Aerospace Electronics Technology, \\ Shanghai, China
}

The polarization-insensitive reflective metasurfaces constructed from hexagonal-ring units along with honeycomb lattice are proposed for the efficient generation of converged orbital angular momentum (OAM) waves. The proposed unit has a very succinct structure constructed from a hexagonal-ring patch along with a hexagonal metallic reflector, which could achieve a polarization-insensitive response to the incident waves, when binding further with the hexagonal lattice. Through the topology optimization of the unit arrangement, two reflective metasurfaces stimulating the first-order and the second-order OAM beams are numerically designed, which may find useful in radio communications and radar applications.

Keywords: OAM, metasurface, reflective, hexagonal-ring, polarization-insensitive

OPEN ACCESS

Edited by:

Weiren Zhu,

Shanghai Jiao Tong University, China

Reviewed by:

Aobo Li,

University of California, San Diego,

United States

Xiong Wang,

ShanghaiTech University, China

*Correspondence:

Xudong Bai

bai_xudong@139.com

Specialty section: This article was submitted to Optics and Photonics, a section of the journal

Frontiers in Physics

Received: 05 May 2020 Accepted: 03 June 2020

Published: 24 July 2020

Citation:

Bai X (2020) Polarization-Insensitive

Reflective Metasurfaces for Highly

Efficient Generation of OAM Beams.

Front. Phys. 8:244.

doi: 10.3389/fphy.2020.00244

\section{INTRODUCTION}

Since the first exploration by Allen et al., orbital angular momentum (OAM) has displayed its independent value in many interdisciplinary fields and has become one of the most important research frontiers in modern technologies [1]. Recently, OAM has been thoroughly studied and widely understood as a promising candidate for information multiplexing to increase the spectral efficiency in microwave domain [2], and the capacity of the wireless communication systems can thus be largely extended [3, 4]. Many researches have then been conducted for stimulating OAM waves, which can generally be split into two categories, the microwave antenna method [5-11] and the physical optics method [12-23].

One of the most elegant approach to stimulate OAM for microwave antenna method is based on high-order mode patch antenna [5]. For example, dual circularly polarized (CP) dual-mode vortex OAM beams were succinctly generated by exciting the second-order resonance modes on a single patch antenna, which is with very compact structure and much suitable for low-cost OAM applications [6]. For more flexible multi-mode OAM modulation, circular phased array systems are constructed to provide the successive phase shifts in elements [7]. The dual-mode OAM operation was realized in a four-element array by employing the opposite phase differences created for left-handed CP and right-handed CP incidences, where the four dual-CP elements are sequentially rotated [8]. To stimulate more OAM modes associated with multi-polarization operation, circular horn array using elements with three polarizations was realized by introducing the phase control networks, which can be very costly and complicated [9]. For the low-cost multi-mode OAM operation, Rotman lens was introduced to simplify the feed network for the phased array [10], and later combined with Fabry-Perot resonant cavity to improve the beam divergence for different OAM modes [11]. Major drawbacks with such microwave antenna methods are that versatile modulations of mixed or multi-OAM modes in different propagation directions are hardly achieved. 
In-depth research and exploration based on physical optics theory have been devoted to stimulate OAM beams through the wavefront transformation of the spherical or plane waves, including the dielectric spiral plate [12], discrete spiral reflector [13], and twisted parabolic reflector [14], whose structures are all very ponderous and enormous in real applications. Recently, metasurfaces have been widely researched for the independent talent of delicate regulation on electromagnetic waves [15-17], which also draw significant attention as a promising realization approach for OAM generation in physical optics method [18-26]. High-efficiency reflective and transmissive metasurfaces with various topological forms have been proposed for vortex OAM generation in linear polarization [18-20]. For the converging OAM radiation with CP waves, broadband transmissive metasurfaces based on PancharatnamBerry phase or photon spin Hall effect have been efficiently demonstrated [21-24]. Recently, to improve the isotropy and spatial coverage property of the metasurfaces, polarizationinsensitive transmissive metasurfaces with honeycomb lattice or rotational symmetry arrangement are proposed to create converging OAM beams for fully polarized applications $[25,26]$, which are troubled with the complex design along with the complicated configurations.

In this paper, we propose the design of polarizationinsensitive reflective metasurfaces with very succinct units along with a honeycomb lattice for the efficient generation of converging OAM waves. The proposed units are constructed from hexagonal-ring patches along with hexagonal metallic reflectors, which could obtain a full-polarization response of the incident wave, when binding further with the hexagonal lattice. Through the topology optimization of the unit arrangement, the reflective metasurfaces stimulating the first-order and the second-order OAM beams are numerically demonstrated.

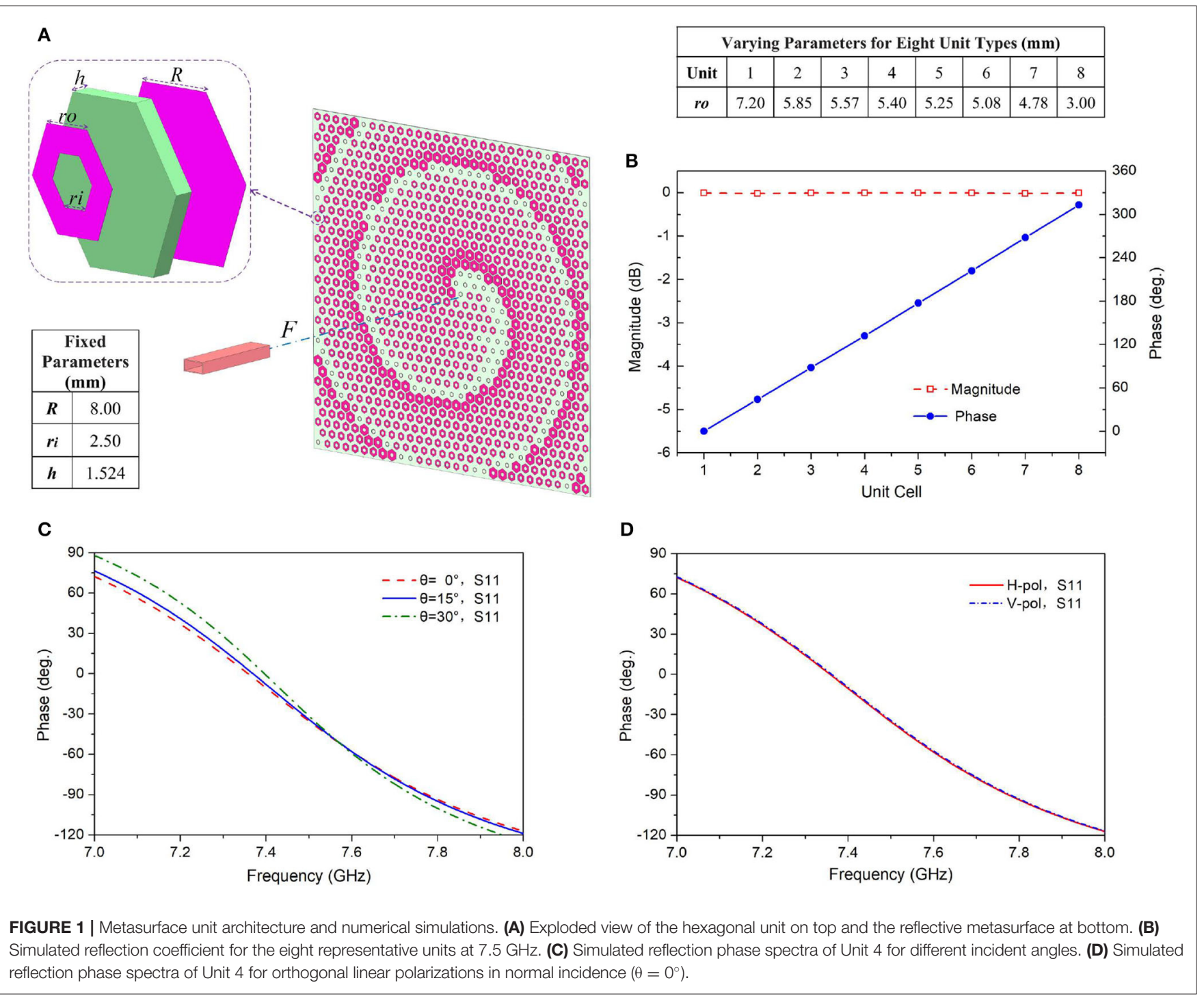




\section{HEXAGONAL UNIT DESIGN}

Figure 1A presents the architecture of the hexagonal units in the metasurface, which have a very succinct structure and composed of two metal layers supported by a substrate layer. The numerical simulations of the units are carried out with the support of CST Microwave Studio Software by using unit-cell boundary conditions along with the Floquet-port excitations. The substrate layer has a dielectric constant of 3.66, a dissipation factor of $\tan \delta=0.0037$, and a thickness of $h=1.524 \mathrm{~mm}$. The unit is a hexagonal structure with a periodic side length $R=8 \mathrm{~mm}$, corresponding to a central working frequency $f=7.5 \mathrm{GHz}$. The upper hexagonal-ring patch serves as the phase-shift metasurface, while the lower metal ground layer plays the role of the metallic reflector. The reflective phase modulation of the units can be achieved by varying the outer side length $r_{o}$ of the hexagonalring patch, while the inner side length $r_{i}$ remains constant.
Based on the former study on the phase-quantization effects for the metasurface lens, the quantization loss for the typical 3bit metasurfaces is only $0.2 \mathrm{~dB}$, which is within the scope of acceptable [25]. Eight representative units are thus selected for the construction of the honeycomb reflectarray to minimize the reflection losses and acquire the high-linearity phase-shift range of $2 \pi$, as shown in Figure 1B. Almost complete reflections have been obtained for all eight representative units, along with the full $2 \pi$ phase modulation. All the optimized parameters for the designed representative units are provided in Figure 1.

Figure 1C presents the simulated reflection phase spectra of Unit 4 for different incident angles as representative, and the phase shift has relatively minor change while varying the incidence angle, implying that the hexagonal unit possesses a good spatial coverage property. Figure 1D shows the simulated phase spectrum in reflection coefficient of Unit 4 for orthogonal linear polarizations as representative, and
A

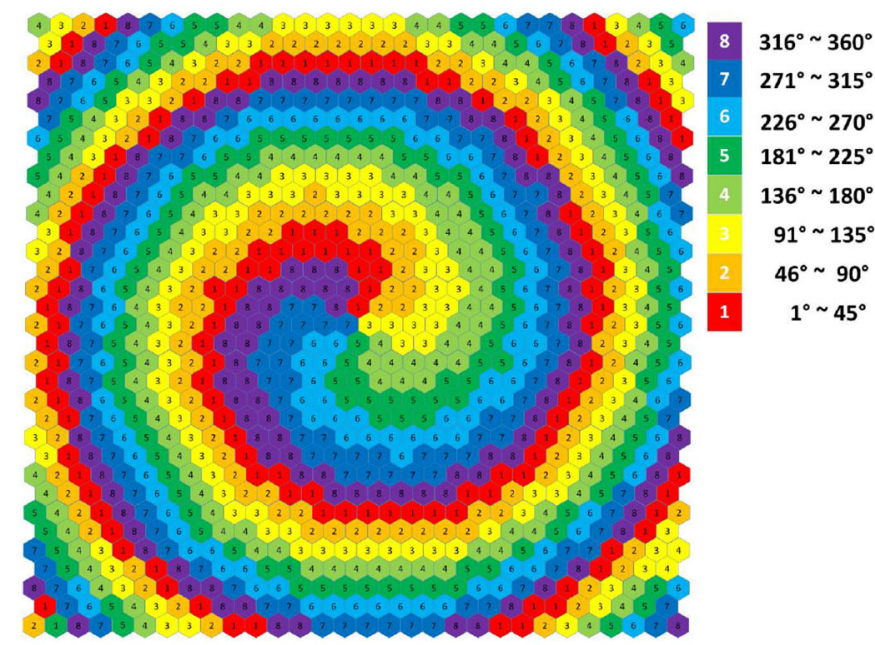

C

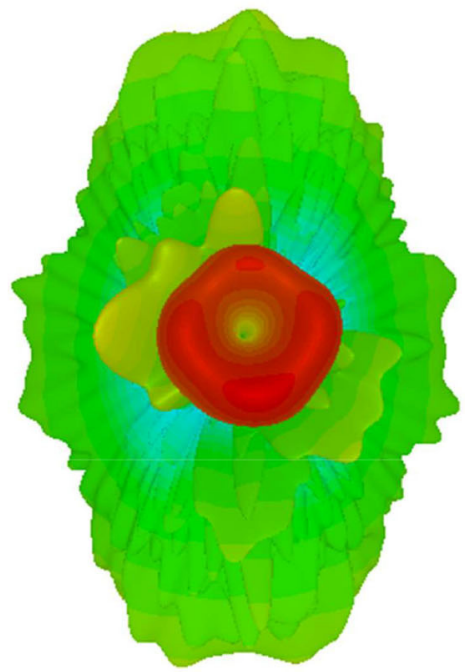

B

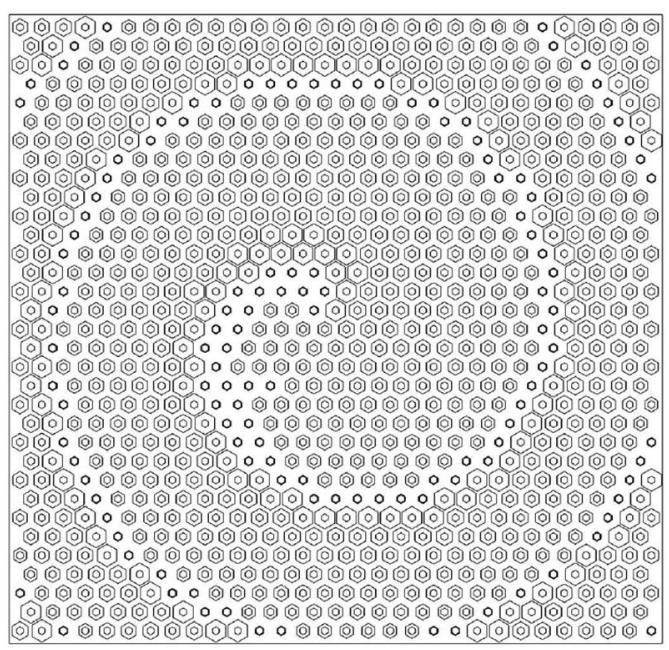

D

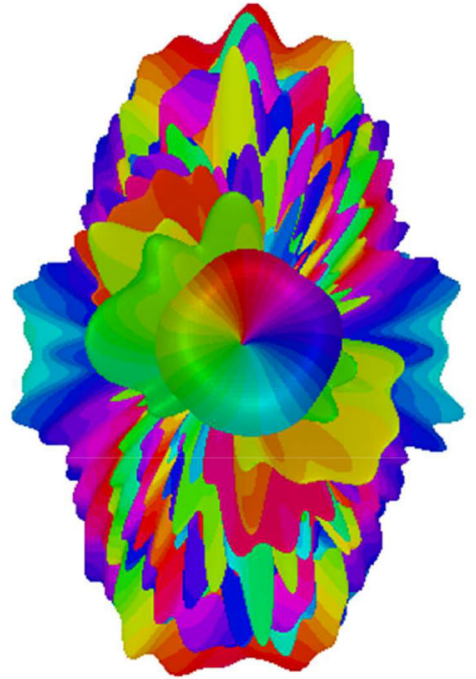

deg.

360

338

315

293

270

248

225

203

180

158

113

67.5

45

22.5

0

FIGURE 2 | Reflective metasurface for OAM mode / = +1. (A) Quantized phase distributions for the units. (B) Topological structure of the metasurface. (C) The amplitude pattern of the simulated far-field radiation. (D) The phase pattern of the simulated far-field radiation. 
almost no phase shifts could be observed for the two different polarizations, implying that the hexagonal unit possesses good polarization-insensitive characteristic.

\section{METASURFACES CONFIGURATION AND VERIFICATION}

The proposed reflective metasurfaces are constructed with the honeycomb lattice by using 974 hexagonal units, and a standard waveguide horn is positioned in the metasurface central axis as the space feed source, as shown in Figure 1A. To improve the beam divergence and obtain the convergent OAM beams through the proposed reflective metasurfaces, the theoretical phase modulation $\phi$ for the metasurface units should be designed to meet the equation below [27]:

$$
\phi=2 \pi\left(\sqrt{\left(x^{2}+y^{2}\right)+F^{2}}-F\right) / \lambda+l \cdot \arctan (y / x)
$$

Here $l$ is the designed OAM mode number, $(x, y)$ are the unit position coordinates, $\lambda$ is the free-space wavelength, and $F$ is the focal length of the feed antenna and set as $F=8 \lambda=320 \mathrm{~mm}$.

To stimulate the OAM wave with mode number $l=+1$, the required phase-shift distribution for all the units are designed using Equation (1) and performed as shown in Figure 2A. The corresponding topological structure of the honeycomb metasurface is provided in Figure 2B, which clearly exhibits a single spiral configuration. Figure $\mathbf{2 C}$ presents the amplitude pattern of the simulated far-field radiation of the honeycomb metasurface for OAM mode number $l=+1$, where an annular tapered pattern of high-intensity radiation is clearly revealed. The side lobes are much lower than the main lobe, indicating that the electromagnetic energy is mainly propagating with the OAM
A

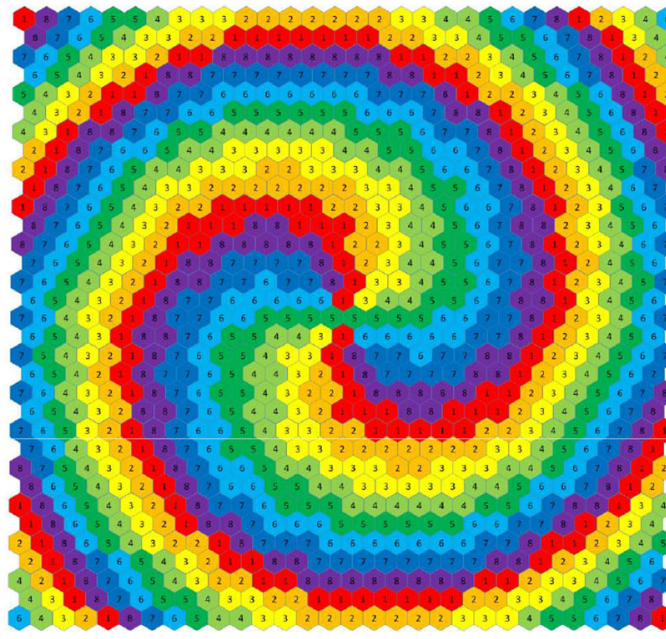

C

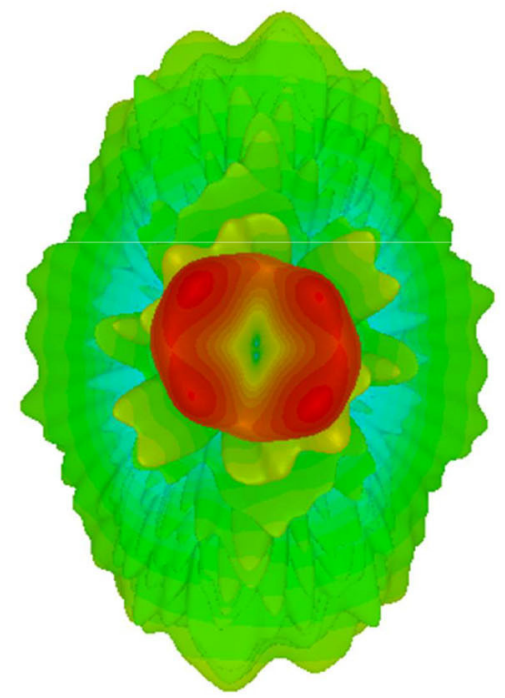

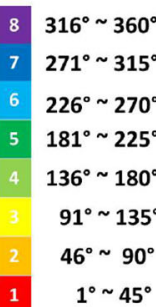

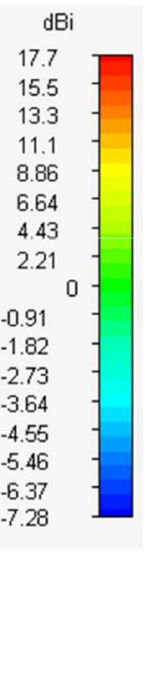

B

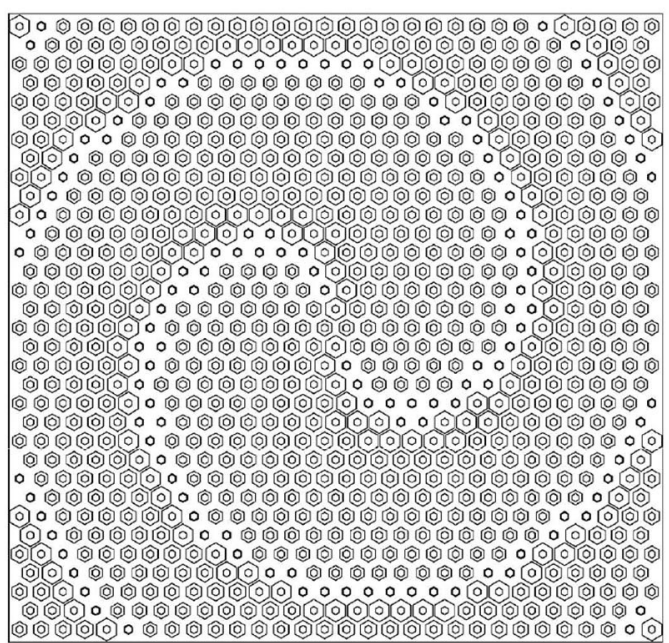

D

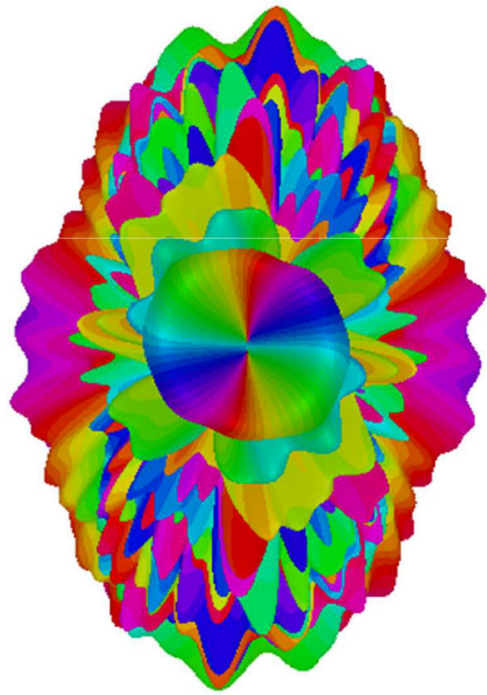

deg.

360

338

315

293

270

248

225

203

180

158

135

113

90

67.5
45

22.5

FIGURE 3 | Reflective metasurface for OAM mode / = +2. (A) Quantized phase distributions for the units. (B) Topological structure of the metasurface. (C) The amplitude pattern of the simulated far-field radiation. (D) The phase pattern of simulated far-field radiation. 
wave. The corresponding phase pattern of the simulated far-field radiation is also plotted in Figure 2D, where the characteristic spiral phase front along with on-axis phase singularity of OAM are clearly revealed. The phase distribution of the OAM waves undergoes a linear variation of $2 \pi$ around the axis of propagation, indicating the creation of a vortex beam with OAM mode $l=+1$.

For the OAM mode with number $l=+2$, the required phaseshift distributions are provided in Figure $\mathbf{3 A}$, along with the corresponding metasurface structure shown in Figure 3B, both clearly exhibiting the double-helix configuration. Figures 3C,D present the amplitude and phase patterns of the simulated farfield radiation for OAM $l=+2$, respectively. The high-intensity annular tapered patterns along with the characteristic spiral phase fronts as well as on-axis phase singularity are clearly exhibited. The phase distribution undergoes a linear variation of $4 \pi$ around the axis of propagation, indicating the creation of a vortex beam with OAM mode $l=+2$.

\section{CONCLUSION}

In summary, polarization-insensitive reflective metasurfaces constructed from hexagonal-ring units along with honeycomb lattice have been proposed for efficient converging OAM

\section{REFERENCES}

1. Allen L, Beijersbergen MW, Spreeuw RJC, Woerdman JP. Optical angular momentum of light and the transformation of Laguerre-Gauss laser modes. Phys Rev A. (1992) 45:8185-9. doi: 10.1103/PhysRev A.45.8185

2. Thidé B, Then H, Sjöholm J, Palmer K, Bergman J, Carozzi TD, et al. Utilization of photon orbital angular momentum in the low-frequency radio domain. Phys Rev Lett. (2007) 99:087701. doi: 10.1103/PhysRevLett.99.087701

3. $\mathrm{Hu} \mathrm{T}$, Wang $\mathrm{Y}$, Liao $\mathrm{X}$, Zhang $\mathrm{J}$, Song Q. OFDM-OAM modulation for future wireless communications. IEEE Access. (2019) 7:59114-25. doi: 10.1109/ACCESS.2019.2915035

4. Guo Z, Yang G. Radial uniform circular antenna array for dual-mode OAM communication. IEEE Anten. Wireless Propag. Lett. (2017) 16:4047. doi: 10.1109/LAWP.2016.2581204

5. Barbuto M, Trotta F, Bilotti F, Toscano A. Circular polarized patch antenna generating orbital angular momentum. Prog Electromagn Res. (2014) 148:2330. doi: 10.2528/PIER14050204

6. Bai X, Liang X, Li M, Zhou B, Geng J, Jin R. Dual-circularly polarized conicalbeam microstrip antenna. IEEE Anten Wireless Propag Lett. (2015) 14:482-5. doi: 10.1109/LAWP.2014.2369515

7. Mohammadi SM, Daldorff LKS, Bergman JES, Karlsson RL, Thidé B, K. Forozesh, et al. Orbital angular momentum in radio-a system study. IEEE Trans Antennas Propag. (2010) 58:565-72. doi: 10.1109/TAP.2009.2037701

8. Bai X, Liang X, Sun Y, Hu P, Yao Y, K. Wang, et al. Experimental array for generating dual-circularly polarized dual-mode OAM radio beams. Sci Rep. (2017) 7:40099. doi: 10.1038/ srep40099

9. Bai X, Jin R, Liu L, Geng J, Liang X. Generation of OAM radio waves with three polarizations using circular horn antenna array. Int J Anten Propag. (2015) 2015:132549. doi: 10.1155/2015/132549

10. Bai X, Liang X, Li J, Wang K, Geng J, Jin R. Rotman lens-based circular array for generating five-mode OAM radio beams. Sci. Rep. (2016) 6:27815. doi: 10.1038/srep27815

11. Bai X, Cao A, Kong F, Qian J, Xu S, Yan W, et al. Rotman lensfed Fabry-Perot resonator antennas for generating converged multi-mode OAM beams. IEEE Access. (2019) 7:105768-75. doi: 10.1109/ACCESS.2019.2 932199 generation. The proposed unit is made of a very succinct structure that binds further with the hexagonal lattice for obtaining a full-polarization response to the incident wave. Through the topology optimization of the units, two reflective metasurfaces stimulating the first-order and the second-order OAM beams have been numerically verified, which could be a promising candidate for radio communications and radar applications.

\section{DATA AVAILABILITY STATEMENT}

The raw data supporting the conclusions of this article will be made available by the author, without undue reservation.

\section{AUTHOR CONTRIBUTIONS}

$\mathrm{XB}$ designed and performed the design and simulation as well as wrote the paper.

\section{FUNDING}

This work was supported by the Shanghai Aerospace PreResearch Foundation (182Y002701).
12. Niemiec R, Brousseau C, Emile O, Ménard A. Characterization of an OAM flat-plate antenna in millimetre frequency band. IEEE Anten Wireless Propag Lett. (2014) 13:1011-4. doi: 10.1109/LAWP.2014.2326525

13. Tamburini F, Mari E, Thidé B, Barbieri C, Romanato F. Experimental verification of photon angular momentum and vorticity with radio techniques. Appl Phys Lett. (2011) 99:204102. doi: 10.1063/1.3659466

14. Mari E, Spinello F, Oldoni M, Ravanelli RA, Romanato F, Parisi G. Nearfield experimental verification of separation of OAM channels. IEEE Antennas Wireless Propag Lett. (2015) 14:556-8. doi: 10.1109/LAWP.2014.2369536

15. Li A, Singh S, Sievenpiper D. Metasurfaces and their applications. Nanophotonics. (2018) 7:989-1011. doi: 10.1515/nanoph-2017-0120

16. Glybovski SB, Tretyakov SA, Belov PA, Kivshar YS, Simovski CR. Metasurfaces: From microwaves to visible. Phys Rep. (2016) 634:172. doi: 10.1016/j.physrep.2016.04.004

17. Li A, Li Y, Long J, Forati E, Du Z, D. Sievenpiper. Time-moduated nonreciprocal metasurface absorber for surface waves. Opt Lett. (2020) 45:1212-5. doi: 10.1364/OL.382865

18. Yu S, Li L, Shi G, Zhu C, Zhou X, Shi Y. Design, fabrication, and measurement of reflective metasurface for orbital angular momentum vortex wave in radio frequency domain. Appl Phys Lett. (2016) 108:121903. doi: 10.1063/1.4944789

19. Akram MR, Ding G, Chen K, Feng Y, Zhu W. Ultra-thin single layer metasurfaces with ultra-wideband operation for both transmission and reflection. Adv Mater. (2020) 32:1907308. doi: 10.1002/adma.201907308

20. Chen Y, Zheng S, Li Y, Hui X, Jin X, H. Chi, et al. A flat-lensed spiral phase plate based on phase-shifting surface for generation of millimeterwave OAM beam. IEEE Anten Wireless Propag Lett. (2016) 15:11568. doi: 10.1109/LAWP.2015.2497243

21. Akram MR, Bai X, Jin R, Vandenbosch GAE, Premaratne M, Zhu W. Photon spin Hall effect based ultra-thin transmissive metasurface for efficient generation of OAM waves. IEEE Trans Antennas Propag. (2019) 67:46508. doi: 10.1109/TAP.2019.2905777

22. Xu H, Liu H, Ling X, Sun Y, Yuan F. Broadband vortex beam generation using multimode Pancharatnam-Berry metasurface. IEEE Trans Antennas Propag. (2017) 65:7378-82. doi: 10.1109/TAP.2017.2761548

23. Zhang K, Yuan Y, Zhang D, Ding X, Ratni B, S. Burokur, et al. Phase-engineered metalenses to generate converging and nondiffractive vortex beam carrying orbital angular momentum in 
microwave region. Opt Express. (2018) 26:1351-60. doi: 10.1364/OE.26.0 01351

24. Akram MR, Mehmood MQ, Bai X, Jin R, Premaratne M, Zhu W. High efficiency ultra-thin transmissive metasurfaces. Adv Opt Mater. (2019) 7:1801628. doi: 10.1002/adom.201801628

25. Bai X, Kong F, Qian J, Song Y, He C, Liang X, et al. Polarizationinsensitive metasurface lens for efficient generation of convergent OAM beams. IEEE Antennas Wireless Propag Lett. (2019) 18:2696-700. doi: 10.1109/LAWP.2019.2949085

26. Akram M, Mehmood M, Tauqeer T, Rana A, Rukhlenko I, Zhu W. Highly efficient generation of Bessel beams with polarization insensitive metasurfaces. Opt Express. (2019) 27:9467-80. doi: 10.1364/OE.27.0 09467
27. Qin F, Wan L, Li L, Zhang H, Wei G, Gao S. A transmission metasurface for generating OAM beams. IEEE Antennas Wireless Propag Lett. (2018) 17:1793-6. doi: 10.1109/LAWP.2018.2867045

Conflict of Interest: XB was employed by the company Shanghai Aerospace Electronics Company Ltd., China Academy of Aerospace Electronics Technology.

Copyright (c) 2020 Bai. This is an open-access article distributed under the terms of the Creative Commons Attribution License (CC BY). The use, distribution or reproduction in other forums is permitted, provided the original author(s) and the copyright owner(s) are credited and that the original publication in this journal is cited, in accordance with accepted academic practice. No use, distribution or reproduction is permitted which does not comply with these terms. 